\begin{tabular}{|c|l|}
\hline Title & $\begin{array}{l}\text { Intracellular local ization and tissue specific distribution of human and yeast DHHC cysteine rich domain-containing } \\
\text { proteins }\end{array}$ \\
\hline Author(s) & Ohno, Y usuke; Kinara, A kio; Sano, Takamitsu; Igarashi, Y asuyuki \\
\hline Citation & $\begin{array}{l}\text { Biochimica et Biophysica A cta (BBA) - Molecular and Cell Biology of Lipids, 1761(4), 474.483 } \\
\text { https://doi.org/10.1016/.bbalip.2006.03.010 }\end{array}$ \\
\hline Issue Date & 2006-04 \\
\hline Doc URL & http://hdl.handle.net/2115/14509 \\
\hline Type & article (author version) \\
\hline File Information & Ohno et al.pdf \\
\hline
\end{tabular}

Instructions for use 


\title{
Intracellular localization and tissue-specific distribution of human and yeast DHHC cysteine-rich domain-containing proteins
}

\section{Yusuke Ohno ${ }^{a}$, Akio Kihara ${ }^{a, "}$, Takamitsu Sano, and Yasuyuki Igarashi}

Department of Biomembrane and Biofunctional Chemistry, Graduate School of

Pharmaceutical Sciences, Hokkaido University, Kita 12-jo, Nishi 6-choume, Kita-ku,

Sapporo 060-0812, Japan

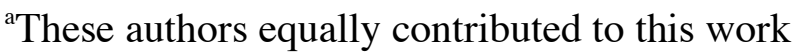

*Corresponding author

Address correspondence to:

\author{
Akio Kihara \\ Department of Biomembrane and Biofunctional Chemistry, \\ Graduate School of Pharmaceutical Sciences, Hokkaido University, \\ Kita 12-jo, Nishi 6-choume, Kita-ku, Sapporo 060-0812, Japan \\ Telephone: +81-11-706-3971 \\ Fax: +81-11-706-4986 \\ E-mail: kihara@pharm.hokudai.ac.jp
}

Keywords: DHHC cysteine-rich domain; Palmitoylation; Protein acyltransferase 


\begin{abstract}
Increasing evidence indicates that DHHC cysteine-rich domain-containing proteins (DHHC proteins) are protein acyltransferases. Although multiple DHHC proteins are found in eukaryotes, characterization has been examined for only a few. Here, we have cloned all the yeast and human $D H H C$ genes and investigated their intracellular localization and tissue-specific expression. Most DHHC proteins are localized in the ER and/or Golgi, with a few localized in the plasma membrane and one in the yeast vacuole. Human $D H H C$ mRNAs also differ in their tissue-specific expression. These results may provide clues to aid in discovering the specific function(s) of each DHHC protein.
\end{abstract}

Abbreviations: ER, endoplasmic reticulum; EGFP, enhanced green fluorescent protein; 3xHA, triple HA; GAPDH, glyceraldehyde 3-phosphate dehydrogenase; HEK, human embryonic kidney; $\mathrm{His}_{6}$, hexa-histidine; PAT, protein acyltransferase; RT, reverse transcription; SC, synthetic complete 


\section{Introduction}

Many proteins, especially those involved in signaling events, are modified by palmitoylation on a cysteine residue. Palmitoylation has been shown to regulate protein stability, intracellular localization, and activities, as well as protein-protein and protein-lipid interactions $[1,2]$. Palmitoylation occurs post-translation and is reversible, implying that the modified proteins are regulated through a palmitoylation-depalmitoylation cycle.

Although palmitoylation was first described over 30 years ago, the responsible genes have only recently been identified. In 2002, yeast genetic approaches identified two DHHC cysteine-rich domain-containing proteins (referred to here DHHC proteins), Akr1 and Erf2, as protein acyltransferases (PATs), with the Cys residue of the DHHC motif forming an acyl-intermediate $[3,4]$. The DHHC cysteine-rich domain is a highly conserved region found in multiple eukarotic proteins ( 7 in yeast and 23 in mouse; Fig. 1) [5]. There is increasing evidence that DHHC proteins other than Akr1 and Erf2 also act as PATs [6-12].

It is unclear why so many DHHC proteins exist in a single organism. One explanation is that each DHHC protein might act on specific substrate(s). Indeed, several yeast genetic studies have demonstrated that each substrate is palmitoylated mainly by a single responsible PAT $[3,4,6,7,13]$. It is also possible that DHHC proteins differ in their intracellular localization or tissue-specific expression patterns. However, only limited information exists regarding these characteristics $[3,6,7,10,12$, 14-18]. Therefore, in the present study we cloned all yeast and human $D H H C$ genes and 
examined their intracellular localization and tissue-specific expression. These analyses revealed that each DHHC protein exhibits a distinguishing localization (ER, Golgi, vacuole, or plasma membrane) and tissue distribution. 


\section{Materials and methods}

\subsection{Yeast strain and media}

The Saccharomyces cerevisiae strains used were SEY6210 [19] and KHY128 [20]. Cells were grown either in YPD medium (1\% yeast extract, $2 \%$ peptone, and $2 \%$ glucose) or in synthetic complete (SC) medium containing nutritional supplements.

\subsection{Cell culture and transfection}

Human embryonic kidney (HEK) 293T cells were grown in Dulbecco's modified Eagle's medium (D6429; Sigma) containing 10\% FCS and supplemented with 100 units/ml penicillin and $100 \mu \mathrm{g} / \mathrm{ml}$ streptomycin, in $0.3 \%$ collagen-coated dishes. Transfections were performed using Lipofectamine Plus ${ }^{\mathrm{TM}}$ Reagent (Invitrogen).

\subsection{Plasmids}

Yeast $2 \mu$-based high-copy-number plasmids encoding C-terminally triple HA (3xHA)-tagged DHHC proteins were used (specifically pAK826, SWF1-3xHA; pAK827, PFA3-3xHA; pAK828, PFA4-3xHA; pAK829, PFA5-3xHA; pAK830, ERF2-3xHA; pAK245, AKR1-3xHA; and pAK247, AKR2-3xHA). The pAK290 plasmid, a derivative of pRS423 (HIS3 marker) [21], was constructed to produce a 3xHA-tagged protein. The yeast $D H H C$ genes were amplified from S288C genomic DNA (Invitrogen) using appropriate primers, as described in Tables 1 and 2. The amplified fragments were cloned into the pAK290 plasmid.

The pUG23 plasmid, a yeast expression vector constructed to produce a fusion 
protein with a C-terminal enhanced green fluorescent protein (EGFP) under the control of the MET25 promoter, was a gift from J. H. Hegemann. The pAK843 (SWF1-EGFP), pAK844 (PFA3-EGFP), pAK845 (PFA4-EGFP), and pAK846 (PFA5-EGFP) plasmids were constructed as follows. The SWF1, PFA3, PFA4, and PFA5 genes were amplified from pAK826, pAK827, pAK828, and pAK829 plasmids, respectively, using appropriate primers (SWF1, SWF1-F2 and SWF1-R1; PFA3, PFA3-F2 and PFA3-R1; PFA4, PFA4-F2 and PFA4-R1; and PFA5, PFA5-F2 and PFA5-R1). The amplified fragments were cloned into the pUG23 plasmid.

Human $D H H C$ genes were amplified using respective primers and templates as described in Tables 1 and 2. The amplified genes were first cloned into the pGEM-T Easy vector (Promega, Madison, WI). Although most of the $D H H C$ genes were obtained as full-length open reading frames, deletion at the 5'-terminus in the $\mathrm{DHHC}-14$ gene and two insertions in the $\mathrm{DHHC}$-20 gene were observed. Therefore, the 5'-terminus of the $\mathrm{DHHC}$ - 14 gene was extended by successive PCR using forward primers (DHHC14-F1, -F2, -F3, F4, and -F5) covering the deleted region with the reverse primer DHHC14-R1. The two insertions found in the $\mathrm{DHHC}-20$ gene were removed by site-directed mutagenesis using a QuikChange site-directed mutagenesis kit (Stratagene, La Jolla, CA) and two primer sets (DHHC20-Q1/Q1c and DHHC20-Q2/Q2c).

Each full-length $\mathrm{DHHC}$ gene was then cloned into the pCE-puro $\mathrm{His}_{6}$-Myc-1 vector, which had been designed to produce an $\mathrm{N}$-terminal hexa-histidine $\left(\mathrm{His}_{6}\right)$ - and Myc- tandemly tagged protein, thus producing pCE-puro $\mathrm{His}_{6}-\mathrm{Myc}-\mathrm{DHHCx}$ plasmid 
(with $\mathrm{x}$ representing the DHHC number). The DHHC-1, -2, -5, -16, -19, and -21 genes were also each cloned into pCE-puro 3xFLAG-1 vector, which had been designed to produce an N-terminal 3xFLAG-tagged protein, to create the respective pCE-puro 3xFLAG-DHHCx plasmid.

\subsection{Reverse transcription (RT)-PCR}

First-strand cDNAs from various human tissues (Human MTC $^{\mathrm{TM}}$ panels I and II) were purchased from TAKARA Bio (Shiga, Japan). Each DHHC cDNA and glyceraldehyde 3-phosphate dehydrogenase (GAPDH) cDNA were amplified by PCR using the tissue cDNAs and primers as described in Table 3.

\subsection{Immunoblotting}

Immunoblotting was performed as described previously [20, 22]. Anti-HA Y-11 (0.2 $\mu \mathrm{g} / \mathrm{ml}$; Santa Cruz Biotechnology, Santa Cruz, CA), anti-FLAG M2 (1 $\mu \mathrm{g} / \mathrm{ml}$; Stratagene, La Jolla, CA), anti-Myc PL14 (1 $\mu \mathrm{g} / \mathrm{ml}$; Medical \& Biological Laboratories, Nagoya, Japan), and actin (1/400 dilution; SIGMA) antibodies were used as primary antibodies. HRP-conjugated anti-mouse or anti-rabbit $\operatorname{IgG~F}(\mathrm{ab})_{2}$ fragment (both from Amersham Biosciences, Piscataway, NJ, and diluted 1:7500) were used as secondary antibodies. Labeling was detected using an $\mathrm{ECL}^{\mathrm{TM}}$ kit (Amersham Biosciences).

\subsection{Fluorescence microscopy}

Immunofluorescence microscopic analyses of cultured cells and yeast were done as 
described previously [20, 23]. Anti-HA Y-11 (2 $\mu \mathrm{g} / \mathrm{ml})$, anti-Myc PL14 (2.5 $\mu \mathrm{g} / \mathrm{ml})$, anti-Myc A-14 (1 $\mu \mathrm{g} / \mathrm{ml}$; Santa Cruz Biotechnology), anti-calreticulin (1/400 dilution; Alexis Biochemicals, Lausen, Switzerland), anti-GM130 (1/100 dilution; BD Biosciences, San Jose, CA), and anti-FLAG M2 (0.5 $\mu \mathrm{g} / \mathrm{ml})$ antibodies were used as primary antibodies. Secondary antibodies included Alexa 488-conjugated anti-rabbit antibody, Alexa 488-conjugated anti-mouse antibody, Alexa 594-conjugated anti-rabbit antibody, and Alexa 594-conjugated anti-mouse antibody (each at $5 \mu \mathrm{g} / \mathrm{ml}$; Invitrogen). Vacuoles in yeast cells were imaged by treatment with $20 \mu \mathrm{M}$ FM4-64, a vacuolar membrane-staining dye (Invitrogen), at $30^{\circ} \mathrm{C}$ for $30 \mathrm{~min}$. Cells were washed with medium, mounted on slides, and analyzed by fluorescence microscopy using an AxioSkop 2 plus microscope (Carl Zeiss, Oberkochen, Germany). 


\section{Results}

\subsection{Intracellular localization of yeast DHHC proteins}

To investigate the localization of yeast DHHC proteins, all yeast $D H H C$ genes ( $W W F 1$, PFA3, PFA4, PFA5, ERF2, AKR1, and AKR2) were cloned and expressed as C-terminally 3xHA-tagged proteins under their own promoters. Pfa3-, Pfa5-, Akr1-, Akr2-, and Erf2-3xHA were readily detected by immunoblotting using anti-HA antibodies (Fig. 2A). However, the amounts of Swf1-3xHA and Pfa4-3xHA were very low. Because these proteins were visualized by the same detection system used for the other proteins, it is reasonable to consider that the detected amounts reflected the strength of their promoters.

Using the same constructs, we performed indirect immunofluorescent microscopic analyses (Fig. 2B). Staining patterns of both Akr1-3xHA and Akr2-3xHA were discontinuous, characteristic of proteins associated with the Golgi apparatus. Erf2-3xHA staining appeared as two ring patterns corresponding to the endoplasmic reticulum (ER), a bright ring around the nucleus (nuclear ER) and a faint one near the cell surface (cortical ER). Pfa3-3xHA was detected in a ring pattern. The rings were determined to correspond to the vacuoles, since the fluorescence of the EGFP-tagged Pfa3 completely merged with the vacuolar membrane-staining dye, FM4-64 (Fig. 3). The localizations of Akr1, Erf2, and Pfa3 were consistent with previous reports [3, 6, 14, $15]$.

We could not detect any fluorescence for Swf1-, Pfa4-, or Pfa5-3xHA using this staining method (data not shown). Therefore, we prepared EGFP fusion constructs 
under an inducible MET25 promoter. Yeast cells expressing EGFP, or Pfa3-, Pfa4-, Pfa5-, or Swf1-EGFP were treated with FM4-64 (Fig. 3). EGFP itself was detected in the cytosol. In contrast, the fluorescence of Pfa3-EGFP merged completely with the FM4-64 staining of the vacuolar membrane, and that of Pfa4-EGFP appeared as two rings, indicative of placement at the ER. The Pfa5-EGFP signal was weak but detectable at the plasma membrane, and in some cells at vacuole-vacuole junctions. Although we still could not detect Swf1-EGFP, even as an EDFP-fusion protein, Swf1 has been reported to be localized in the ER [7]. Altogether, these results indicate that yeast DHHC proteins differ in their intracellular localization.

\subsection{Intracellular localization of human DHHC proteins}

We searched for $D H H C$ genes in the human gene database. Although 23 mouse $D H H C$ genes (DHHC-1 to -23) have been reported [8], in humans the DHHC-23 gene is missing. Therefore, we cloned the other 22 DHHC genes (DHHC-1 to -22) and expressed them in HEK 293 T cells as $\mathrm{N}$-terminally $\mathrm{His}_{6}$-Myc-tagged proteins. Cell lysates were subjected to immunoblotting with an anti-Myc antibody (Fig. 4A). Although most of the DHHC proteins were substantially detectable, DHHC-1, -5, -16, -19 , and -21 were not or only faintly detected. Therefore, constructs of these genes, and DHHC-2 for comparison, were prepared for expression in cells as N-terminally 3xFLAG-tagged proteins. In immunoblots using an anti-FLAG antibody, DHHC-1, -5 , -19 , and -21 were readily detectable, although DHHC-16 was still only faintly detected (Fig. 4B). 
We next investigated the intracellular localization of the $\mathrm{His}_{6}$-Myc-DHHC or 3xFLAG-DHHC proteins by indirect immunofluorescence microscopy (Fig. 5). DHHC-1, -6, -10, -11, -13, -14, and -19 exhibited reticular staining patterns characteristic of ER proteins. We indeed confirmed such localization by overlapping the image with the staining pattern of calreticulin, an ER-resident protein (Fig.6 and data not shown). Aberrant ER structures were observed in some DHHC-6, -10, or -11 -expressing cells, probably due to artificial effects of the overproduction of these proteins. A bright, round ER was detected in the DHHC-6 -expressing cells, whereas the ER was condensed in the DHHC-10 or DHHC-11 -expressing cells (data not shown).

DHHC-3, $-4,-7,-8,-15,-17$, and -18 exhibited perinuclear staining patterns, indicative of placement at the Golgi apparatus (Fig. 5). We confirmed such co-localization by also staining the Golgi marker GM130 (Fig. 6 and data not shown). DHHC-2, -9, -12, and -22 were detected both in the ER and the Golgi (Figs. 5 and 6). Thus, most of the DHHC proteins were localized in the ER and/or Golgi, except DHHC-5, -20, and -21, which were localized in the plasma membrane (Fig. 5), although ER staining was also observed for DHHC-21. We could not determine the localization of DHHC-16, which was only weakly detected by immunoblotting. Reports indicate, however, that DHHC-16 is localized in the ER [18].

\subsection{Tissue-specific distribution of human DHHC mRNAs}

Until now, tissue-specific expression had been examined for only a limited number of 
DHHC genes [8, 12, 16-18, 24, 25]. We investigated the tissue-specific expression of human $D H H C$ mRNAs by RT-PCR. As shown in Fig. 7, DHHC-4, -5, -7, -8, -10, -12, $-13,-17$, and -22 mRNAs were highly ubiquitous. Although $\mathrm{DHHC}-3,-6,-9,-14,-16$, -18 , and -21 mRNAs were also expressed in most tissues examined, they were absent or low in one or more specific tissue. The expression of $D H H C-11,-19$, or -20 mRNA was highly tissue-specific. $D H H C$ - 11 mRNA was detected only in testis, whereas $D H H C-19$ mRNA was highly expressed in testis but weakly in thymus and small intestine. DHHC-20 mRNA was observed in the placenta and testis, with weak expression detected in heart, lung, liver, and thymus. DHHC-2 and -15 mRNAs were almost absent in all tissues tested. Only faint expression was observed in kidney and testis for DHHC-2 mRNA and in heart, brain, lung, kidney, thymus, and small intestine for DHHC-15 mRNA. In conclusion, each $D H H C$ mRNA exhibits a characteristic tissue-specific expression pattern. 


\section{Discussion}

In the present study we investigated the intracellular localization of yeast and human DHHC proteins and found that each protein exhibits a characteristic localization (Table 5). The most frequent organelles in which DHHC proteins were localized included the ER and the Golgi apparatus. In contrast, Pfa5 and DHHC-5, -20, and -21 were localized at the plasma membrane. Yeast Pfa3 alone was localized in the vacuole, and no human DHHC protein was found to be localized in the equivalent mammalian organelle, the lysosome. Since we were detecting overproduced but not endogenous proteins, the possibility cannot be excluded that overproduction caused spill over of some proteins into other organelles. Therefore, preparation of antibodies against each DHHC protein and their usage in localization studies will be required. However, our methods may have an advantage in elucidating specific functions for each DHHC protein.

Palmitoylation is a dynamic process, and several proteins, especially those involved in signal transduction, such as the heterotrimeric $G$ protein $G_{s} \alpha$, the $\beta_{2}$-adrenergic receptor, and endothelial nitric oxide synthase, undergo an acylated-deacylated cycle [26-28]. The plasma membrane-localized Pfa5 and DHHC-5, -20 , and -21 proteins are candidates for PATs involved in this dynamic cycle. On the other hand, the ER- and/or Golgi-localized DHHC proteins may act on de novo synthesized proteins in stimulus-independent processes such as membrane localization and delivery to organelles.

Using their tissue-specific mRNA expression patterns (Fig. 7), we classified the DHHC genes into roughly three groups: highly ubiquitous, nearly ubiquitous, and 
tissue-specific. The highly ubiquitous and nearly ubiquitous groups each include many genes $($ DHHC-4 $,-5,-7,-8,-10,-12,-13,-17$, and -22; and DHHC-1, -3, -6, -9, - 14, -16, $-18,-20$, and -21 , respectively), indicating that multiple DHHC proteins are expressed in the same cell. On the other hand, relatively small numbers of genes are tissue-specific (DHHC-2, -11, -15,-19, and -20). DHHC-11 and -19 mRNAs were specific to testis (Fig. 7). Substantial expression of $D H H C-20$ mRNA was observed in placenta and testis, although weak expression was also detected in other tissues. Only faint expression in some tissues was observed for $D H H C-2$ or -15 mRNA. It is possible that these genes are expressed in specific tissues that were not examined in this study.

Occasionally, two amplified bands were observed for a $D H H C$ mRNA (Fig. 7); the most apparent example being $D H H C-7$ mRNA. We cloned the upper band and found that it represented an alternative splicing isoform that contains an additional $111 \mathrm{bp}$ (data not shown). Since this isoform was expressed in specific tissues such as placenta, lung, liver, thymus, and small intestine, it is possible that it possesses tissue-specific functions.

Recent reports have indicated that some $D H H C$ genes are related to certain pathologies: $D H H C-2$, to a colorectal cancer, a hepatocellular carcinoma, and a nonsmall cell lung cancer [24]; $D H H C-8$, to schizophrenia [29]; and $D H H C-15$, to X-linked mental retardation [30]. However, the substrates of these proteins are unclear at present. It has been suggested that DHHC proteins differ in substrate specificity with some overlap [8]. Therefore, future studies are required to elucidate the specific substrate(s) and physiological/pathological function(s) of each DHHC protein. 


\section{Acknowledgments}

We thank Dr. J. H. Hagemann (Heinrich-Heine University, Düsseldorf, Germany) for kindly providing the plasmid pUG23. This work was supported by grants from the Akiyama Foundation. 


\section{References}

[1] J.T. Dunphy, M.E. Linder, Signalling functions of protein palmitoylation, Biochim. Biophys. Acta 1436 (1998) 245-261.

[2] M.D. Resh, Fatty acylation of proteins: new insights into membrane targeting of myristoylated and palmitoylated proteins, Biochim. Biophys. Acta 1451 (1999) $1-16$.

[3] A.F. Roth, Y. Feng, L. Chen, N.G. Davis, The yeast DHHC cysteine-rich domain protein Akrlp is a palmitoyl transferase, J. Cell Biol. 159 (2002) 23-28.

[4] S. Lobo, W.K. Greentree, M.E. Linder, R.J. Deschenes, Identification of a Ras palmitoyltransferase in Saccharomyces cerevisiae, J. Biol. Chem. 277 (2002) 41268-41273.

[5] T. Putilina, P. Wong, S. Gentleman, The DHHC domain: a new highly conserved cysteine-rich motif, Mol. Cell. Biochem. 195 (1999) 219-226.

[6] J.E. Smotrys, M.J. Schoenfish, M.A. Stutz, M.E. Linder, The vacuolar DHHC-CRD protein Pfa3p is a protein acyltransferase for Vac8p, J. Cell Biol. 170 (2005) 1091-1099.

[7] J. Valdez-Taubas, H. Pelham, Swf1-dependent palmitoylation of the SNARE Tlg1 prevents its ubiquitination and degradation, EMBO J. 24 (2005) 2524-2532.

[8] M. Fukata, Y. Fukata, H. Adesnik, R.A. Nicoll, D.S. Bredt, Identification of PSD-95 palmitoylating enzymes, Neuron 44 (2004) 987-996.

[9] C.E. Ducker, E.M. Stettler, K.J. French, J.J. Upson, C.D. Smith, Huntingtin interacting protein 14 is an oncogenic human protein: palmitoyl acyltransferase, 
Oncogene 23 (2004) 9230-9237.

[10] C.A. Keller, X. Yuan, P. Panzanelli, M.L. Martin, M. Alldred, M. Sassoe-Pognetto, B. Luscher, The $\gamma 2$ subunit of $\mathrm{GABA}_{\mathrm{A}}$ receptors is a substrate for palmitoylation by GODZ, J. Neurosci. Res. 24 (2004) 5881-5891.

[11] K. Huang, A. Yanai, R. Kang, P. Arstikaitis, R.R. Singaraja, M. Metzler, A. Mullard, B. Haigh, C. Gauthier-Campbell, C.A. Gutekunst, M.R. Hayden, A. El-Husseini, Huntingtin-interacting protein HIP14 is a palmitoyl transferase involved in palmitoylation and trafficking of multiple neuronal proteins, Neuron 44 (2004) 977-986.

[12] J.T. Swarthout, S. Lobo, L. Farh, M.R. Croke, W.K. Greentree, R.J. Deschenes, M.E. Linder, DHHC9 and GCP16 constitute a human protein fatty acyltransferase with specificity for H- and N-Ras, J. Biol. Chem. 280 (2005) 31141-31148.

[13] A. Kihara, F. Kurotsu, T. Sano, S. Iwaki, Y. Igarashi, Long-chain base kinase Lcb4 is anchored to the membrane through its palmitoylation by Akr1, Mol. Cell. Biol. 25 (2005) 9189-9197.

[14] P. Babu, R.J. Deschenes, L.C. Robinson, Akr1p-dependent palmitoylation of Yck2p yeast casein kinase 1 is necessary and sufficient for plasma membrane targeting, J. Biol. Chem. 279 (2004) 27138-27147.

[15] D.J. Bartels, D.A. Mitchell, X. Dong, R.J. Deschenes, Erf2, a novel gene product that affects the localization and palmitoylation of Ras2 in Saccharomyces cerevisiae, Mol. Cell. Biol. 19 (1999) 6775-6787.

[16] T. Harada, O. Matsuzaki, H. Hayashi, S. Sugano, A. Matsuda, E. Nishida, AKRL1 
and AKRL2 activate the JNK pathway, Genes Cells 8 (2003) 493-500.

[17] R.R. Singaraja, S. Hadano, M. Metzler, S. Givan, C.L. Wellington, S. Warby, A. Yanai, C.A. Gutekunst, B.R. Leavitt, H. Yi, K. Fichter, L. Gan, K. McCutcheon, V. Chopra, J. Michel, S.M. Hersch, J.E. Ikeda, M.R. Hayden, HIP14, a novel ankyrin domain-containing protein, links huntingtin to intracellular trafficking and endocytosis, Hum. Mol. Genet. 11 (2002) 2815-2828.

[18] B. Li, F. Cong, C.P. Tan, S.X. Wang, S.P. Goff, Aph2, a protein with a $z$-DHHC motif, interacts with c-Abl and has pro-apoptotic activity, J. Biol. Chem. 277 (2002) 28870-28876.

[19] J.S. Robinson, D.J. Klionsky, L.M. Banta, S.D. Emr, Protein sorting in Saccharomyces cerevisiae: isolation of mutants defective in the delivery and processing of multiple vacuolar hydrolases, Mol. Cell. Biol. 8 (1988) 4936-4948.

[20] A. Kihara, Y. Igarashi, Identification and characterization of a Saccharomyces cerevisiae gene, RSB1, involved in sphingoid long-chain base release, J. Biol. Chem. 277 (2002) 30048-30054.

[21] T.W. Christianson, R.S. Sikorski, M. Dante, P. Hieter, Multifunctional yeast high-copy-number shuttle vectors, Gene 110 (1992) 119-122.

[22] A. Kihara, M. Ikeda, Y. Kariya, E. Y. Lee, Y.M. Lee, Y. Igarashi, Sphingosine-1-phosphate lyase is involved in the differentiation of F9 embryonal carcinoma cells to primitive endoderm, J. Biol. Chem. 278 (2003) 14578-14585.

[23] C. Ogawa, A. Kihara, M. Gokoh, Y. Igarashi, Identification and characterization of a novel human sphingosine-1-phosphate phosphohydrolase, hSPP2, J. Biol. 
Chem. 278 (2003) 1268-1272.

[24] T. Oyama, Y. Miyoshi, K. Koyama, H. Nakagawa, T. Yamori, T. Ito, H. Matsuda, H. Arakawa, Y. Nakamura, Isolation of a novel gene on 8p21.3-22 whose expression is reduced significantly in human colorectal cancers with liver metastasis, Genes Chromosomes Cancer 29 (2000) 9-15.

[25] F. Saitoh, Q.B. Tian, A. Okano, H. Sakagami, H. Kondo, T. Suzuki, NIDD, a novel DHHC-containing protein, targets neuronal nitric-oxide synthase (nNOS) to the synaptic membrane through a PDZ-dependent interaction and regulates nNOS activity, J. Biol. Chem. 279 (2004) 29461-29468.

[26] S.M. Mumby, C. Kleuss, A.G. Gilman, Receptor regulation of G-protein palmitoylation, Proc. Natl. Acad. Sci. USA 91 (1994) 2800-2804.

[27] T.P. Loisel, L. Adam, T.E. Hebert, M. Bouvier, Agonist stimulation increases the turnover rate of $\beta_{2} \mathrm{AR}$-bound palmitate and promotes receptor depalmitoylation, Biochemistry 35 (1996) 15923-15932.

[28] L.J. Robinson, L. Busconi, T. Michel, Agonist-modulated palmitoylation of endothelial nitric oxide synthase, J. Biol. Chem. 270 (1995) 995-998.

[29] J. Mukai, H. Liu, R.A. Burt, D.E. Swor, W.S. Lai, M. Karayiorgou, J.A. Gogos, Evidence that the gene encoding ZDHHC8 contributes to the risk of schizophrenia, Nat. Genet. 36 (2004) 725-731.

[30] M.R. Mansouri, L. Marklund, P. Gustavsson, E. Davey, B. Carlsson, C. Larsson, I. White, K.H. Gustavson, N. Dahl, Loss of ZDHHC15 expression in a woman with a balanced translocation $\mathrm{t}(\mathrm{X} ; 15)(\mathrm{q} 13.3 ;$ cen $)$ and severe mental retardation, Eur. J. 
Hum. Genet. 13 (2005) 970-977.

[31] M.G. Claros, G. von Heijne, TopPred II: an improved software for membrane protein structure predictions, Comput. Appl. Biosci. 10 (1994) 685-686. 


\section{Figure legends}

Fig. 1. Structures and dendrogram of DHHC proteins. (A) Structures of human and yeast DHHC proteins are depicted. Pink and red boxes indicate DHHC cysteine-rich domains and ankyrin motifs, respectively. The positions of the transmembrane domains were predicted by the TopPred II 1.1 program [31]. Since regions with a score of more than 1.0 are systematically shown as green boxes, these do not always represent true transmembrane domains. (B) Dendrogram of human and yeast DHHC proteins. Human and yeast DHHC proteins are shown in blue and red, respectively. The GenBank accession numbers are listed in Table 4.

Fig. 2. Expression and localization of HA-tagged yeast DHHC proteins. (A) Total lysates were prepared from SEY6210 cells bearing plasmids encoding no protein or Swf1-, Pfa3-, Pfa4-, Pfa5-, Erf2-, Akr1-, or Akr2-3xHA, as indicated. The HA-tagged proteins were detected by immunoblotting with anti-HA antibodies. Uniform protein loading was demonstrated by immunoblotting with an anti-Pgk1 antibody. (B) KHY128 cells harboring plasmids encoding Akr1-, Akr2-, Erf2-, or Pfa3-3xHA were fixed by formaldehyde, converted to spheroplasts, and permeabilized with $0.1 \%$ Triton $\mathrm{X}-100$. The cells were then stained with anti-HA antibodies (left panels) and 4',6-diamidino-2-phenylindole (DAPI) (middle panels), and the staining patterns compared in merged images (right panels). Bar, $3 \mu \mathrm{m}$.

Fig. 3. Localization of EGFP-fused yeast DHHC proteins. SEY6210 cells bearing 
plasmids encoding EGFP, or Pfa3-, Pfa4-, or Pfa5-EGFP were cultured for $5 \mathrm{~h}$ at $30^{\circ} \mathrm{C}$ in SC medium lacking both histidine and methionine. After a 30 min treatment with 20 $\mu \mathrm{M}$ FM4-64 at $30^{\circ} \mathrm{C}$, cells were analyzed by fluorescence microscopy, AxioSkop 2 plus. Magnification, X 630; bar, $3 \mu \mathrm{m}$.

Fig. 4. Expression of human DHHC proteins. HEK 293T cells were transfected with plasmids encoding the indicated $\mathrm{His}_{6}$-Myc-tagged (A) or 3xFLAG-tagged (B) human DHHC proteins. Total cell lysates were prepared from each culture, separated by SDS-PAGE, and subjected to immunoblotting with anti-Myc, anti-FLAG, or anti-actin antibodies.

Fig. 5. Intracellular localization of human DHHC proteins. HEK 293T cells were transfected with plasmid encoding DHHC-1 through -12 (A) or DHHC-13 through -22 (B), each tagged with His $_{6}$-Myc (DHHC-2, -3, -4, -6, -7, -8, -9, -10, -11, -12, -13, -14, $-15,-17,-18,-20$, or -22 ) or $3 x$ FLAG (DHHC-1, $-5,-19$ or -21$)$. The transfected cells were fixed by formaldehyde, permeabilized with $0.1 \%$ Triton X-100, stained with anti-Myc or anti-FLAG antibodies, and imaged by fluorescent (left panels) or phase contrast (right panels) microscopy. Magnification, X 630; bar, $10 \mu \mathrm{m}$.

Fig. 6. Localization of DHHC-9, $-11,-12,-15$, and -22 in the ER and/or Golgi. HEK $293 \mathrm{~T}$ cells were transfected with plasmids encoding DHHC-9, $-11,-12,-15$, or -22 , each tagged with $\mathrm{His}_{6}$-Myc. The transfected cells were fixed by formaldehyde, 
permeabilized with $0.1 \%$ Triton X-100, double stained with anti-Myc (left panels) and either anti-calreticulin or anti-GM130 (center panels) antibodies. Merged images are presented in the right panels. Magnification, X 630; bar, $10 \mu \mathrm{m}$.

Fig. 7. Tissue-specific expression patterns of human $D H H C$ mRNAs. Using specific primers (listed in Table 3) and PCR, mRNAs of DHHC-1 through -22 and GAPDH were amplified from human tissue cDNAs or from plasmids encoding each $D H H C$ gene. 
Table 1. Primers used in this study

Primer Nucleotide sequence

\begin{tabular}{|c|c|}
\hline SWF1-F1 & 5'-CTCGAGACGGTACTGAAGACAATCCGCTC-3' (XhoI) \\
\hline SWF1-F2 & 5'-ACTAGTAAAGAACAAACAATGTCATGGAATC-3' (SpeI) \\
\hline SWF1-R1 & 5'-ACTAGTTATTAAATCTGTGAGATTGGCCAGG-3' (SpeI) \\
\hline PFA3-F1 & 5'-CTCGAGTTGATATTGCAGAAGATTTCACTG-3' (XhoI) \\
\hline PFA3-F2 & 5'-ACTAGTCTTTTTCGCAAATGAATGACAGG-3' (SpeI) \\
\hline PFA3-R1 & 5'-ACTAGTATTTGCATCGATAATTTCTACAGACG-3' (SpeI) \\
\hline PFA4-F1 & 5'-CTCGAGTCACTCCTTAAGAATTGCTACC-3' (XhoI) \\
\hline PFA4-F2 & 5'-TCTAGATCGTTAAACC ATGCCAGTAAAG-3' (XbaI) \\
\hline PFA4-R1 & 5'-TCTAGATTCC ATATCAACATCCACTCCAAAATCG-3' (XbaI) \\
\hline PFA5-F1 & 5'-CTCGAGTCGCTTCTTCAATTTCATCTCC-3' (XhoI) \\
\hline PFA5-F2 & 5'-ACTAGTTGTATATGAATGGCTCTATC ATGG-3' (SpeI) \\
\hline PFA5-R1 & 5'-ACTAGTGTAAGCAGGATCACTATCGTCACC-3' (SpeI) \\
\hline ERF2-F1 & 5'-CTCGAGCTTGTGTCGCAGAAAATGGAAACG-3' (XhoI) \\
\hline ERF2-R1 & 5'-CCTAGGTATTTTCTGTATTTTTTCAAAGCTGTG-3' (AvrII) \\
\hline AKR1-F1 & 5'-TGAGCCGTCCTGCAAGTCTGGTACC-3' \\
\hline AKR1-R1 & 5'-ACTAGTTACC ATATC ATTTCCTTGTTC-3' (SpeI) \\
\hline AKR2-F1 & 5'-ACCACGATTCCATTGATAGCTAAGG-3' \\
\hline AKR2-R1 & 5'-ACTAGTGTTTGGACGTAATACTTCTCC-3' (SpeI) \\
\hline
\end{tabular}




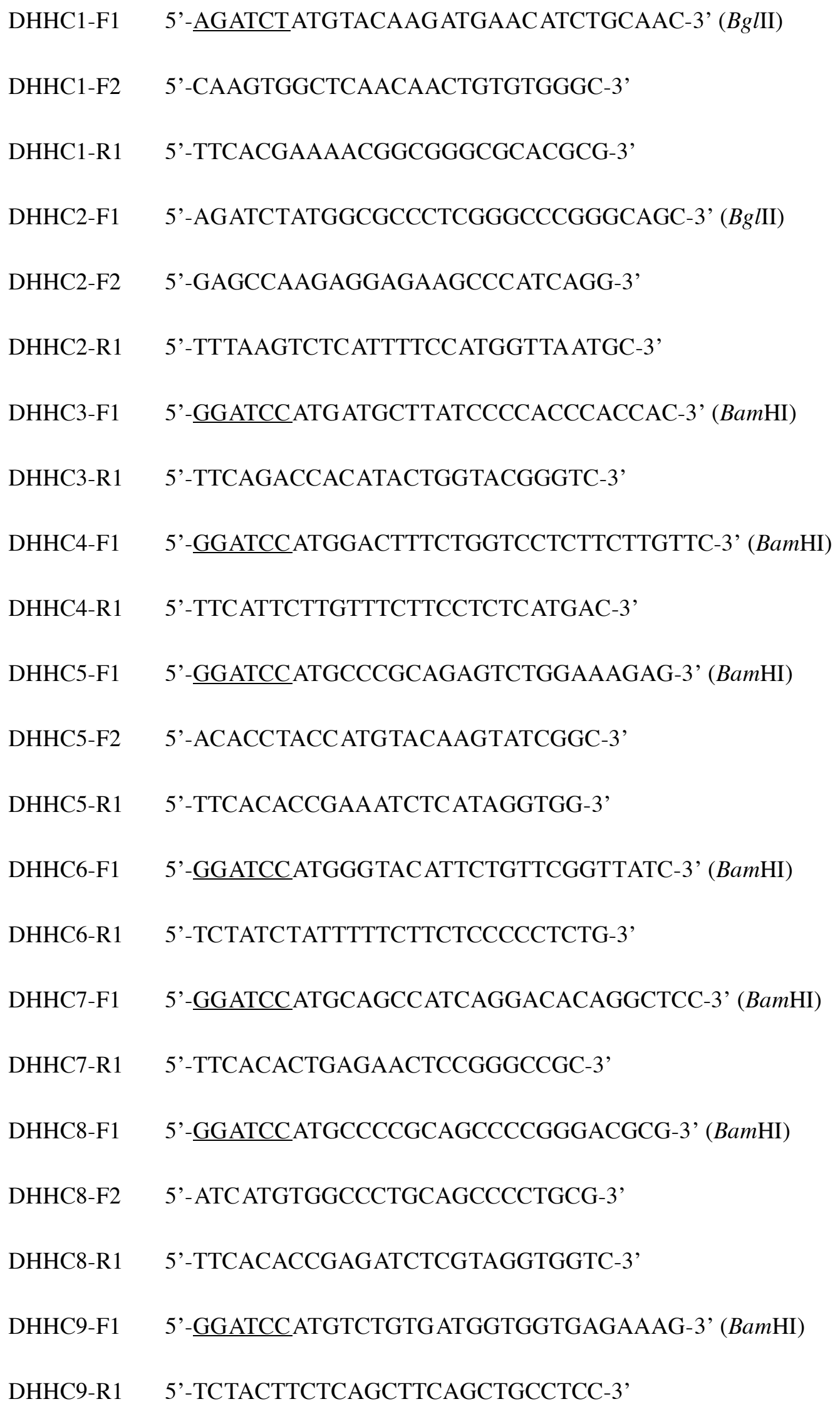


DHHC10-F1 5'-GGATCCATGGACACCCGCTCCGGGAGCCAG-3' (BamHI)

DHHC10-R1 5'-TTCAGTCTTCACTTTCAGCACTGTCAG-3'

DHHC11-F1 5'-GGATCCATGACACAGAAGGGCAGTATGAAGC-3' (BamHI)

DHHC11-R1 5'-TTCATCCAAGTAACCCTGTGTCCACG-3'

DHHC12-F1 5'-GGATCCATGGCGCCCTGGGCGCTCCTCAGCC-3' (BamHI)

DHHC12-R1 5'-TCTAAACAGCTGGGCTGCTGCCCTC-3'

DHHC13-F1 5'-AGATCTATGGGGCAGCCCTGGGCGGCTGGG-3' (BglII)

DHHC13-R1 5'-TTCAGGAGGCTGTGTGTCCCACATCTG-3'

DHHC14-F1 5'-AGATCTATGCCTCCCGGCGGCGGCGGGCCCATGAAAGACTGCGA

GTACAGCCAGATCAGCACCCACAGCTCCTCCCCCATGGAGTCGCCCC ACA-3' (BglII)

DHHC14-F2 5'-CCCCATGGAGTCGCCCCACAAGAAGAAGAAAATCGCGGCCCGGA GGAAATGGGAGGTGTTCCCGGGAAGAAACAAGTTCTTCTGTAACGG GAGG-3'

DHHC14-F3 5'-AGTTCTTCTGTAACGGGAGGATC ATGATGGCCCGGCAGACGGGC GTCTTCTACCTGACGCTCGTCCTCATCCTGGTCACTAGCGGACTCTTC TTC-3

DHHC14-F4 5'-TCACTAGCGGACTCTTCTTCGCCTTCGACTGTCCGTACCTGGCGGT GAAAATCACCCCTGCCATCCCTGCAGTCGCTGGCATCCTGTTCTTCT TTG-3,

DHHC14-F5 5'-CTGGCATCCTGTTCTTCTTTGTGATGGGGACCCTGCTCCGCACCAG CTTCAGCGACCCCGGAGTCCTCCCACGAGCCACGCCTGATGAAGCC 
GCC-3,

DHHC14-F6 5'-CTTTATATTTGC ATTCGTTATCACCCACG-3'

DHHC14-R1 5'-TTCACACGGAGCTGAGCTTCACCAGG-3'

DHHC15-F1 5' -GGATCCATGCGGCGAGGCTGGAAGATGGCTC-3' (BamHI)

DHHC15-F2 5'-AGAAAGACCTGAGGTCCAGAAGCAG-3'

DHHC15-R1 5'-TCTATGTTTCCGTTTCCACAGCAAGAG-3'

DHHC16-F1 5'-GGATCCATGCGAGGCCAGCGGAGCCTGCTGC-3' (BamHI)

DHHC16-R1 5'-TTCACACTGCCATCACAGAGGCTGAG-3'

DHHC17-F1 5'-AGATCTATGCAGCGGGAGGAGGGATTTAAC-3' (BglII)

DGGC17-F2 5'-GAAGCTGGAGCTAATGTTGATGCCC-3'

DHHC17-R1 5'-GTCGACCTACACCAGCTGGTACCCAGATCC-3'

DHHC18-F1 5'-AGATCTATGAAGGACTGCGAGTACCAGCAG-3' (BglII)

DHHC18-F2 5'-GATCTGCTTCTTCTCCATCTGG-3'

DHHC18-R1 5' '-AGATCTTCAGGGGTGGCCTCCTACCATGCTG-3' (BglII)

DHHC19-F1 5' '-GGATCCATGACACTCTTAACGGATGCCACGC-3' (BamHI)

DHHC19-R1 5'-TTCAGCTTGGGAAGCACACGGCCCAG-3'

DHHC20-F1 5'-AGATCTATGGCGCCCTGGACGCTGTGGCGC-3' (BglII)

DHHC20-F2 5'-TGAGAAGAGCAGCAAGAGCTTTACC-3'

DHHC20-R1 5'-TTCATACACCTGATTTGACGATGCC-3'

DHHC20-Q1 5'-CTATACCACATCAGCTTCAAAAACTATCAGATATTGTGAAAAATG $-3$

DHHC20-Q1c 5'-CATTTTTCACAATATCTGATAGTTTTTGAAGCTGATGTGGTATAG- 
DHHC20-Q2 5'-CAGAATGAGTATGCCAGAAGTGGCTCAAATCAACCTTTTCCTATC$3^{\prime}$

DHHC20-Q2c 5'-GATAGGAAAAGGTTGATTTGAGCCACTTCTGGCATACTCATTCTG3 ,

DHHC21-F1 5'-GGATCCATGGGTCTCCGGATTCACTTTGTTG-3' (BamHI)

DHHC21-R1 5'-TTTAGACATGATTGGCAAAGTGGTAGG-3'

DHHC22-F1 5'-GGATCCATGGAGGGGCCGGGGCTGGGCTC-3' (BamHI)

DHHC22-F2 5'-CAGAAGCCAAAATGAGAGCCAACC-3'

DHHC22-R1 5'-TTTTCTTCATACTGAGCGAAGAACC-3'

GAPDH-F1 5'-TGAAGGTCGGAGTCAACGGATTTGGT-3'

GAPDH-R1 5'-CATGTGGGCCATGAGGTCCACCAC-3'

The restriction sites created are underlined. 
Table 2. Templates and primers used for amplifying DHHC genes

Gene Template Primer

\begin{tabular}{|c|c|c|}
\hline$A K R 1$ & S288C genomic DNA & AKR1-F1 and AKR1-R1 \\
\hline$A K R 2$ & S288C genomic DNA & AKR2-F1 and AKR2-R1 \\
\hline$S W F 1$ & S288C genomic DNA & SWF1-F1 and SWF1-R1 \\
\hline PFA3 & S288C genomic DNA & PFA3-F1 and PFA3-R1 \\
\hline PFA4 & S288C genomic DNA & PFA4-F1 and PFA4-R1 \\
\hline PFA5 & S288C genomic DNA & PFA5-F1 and PFA5-R1 \\
\hline$E R F 2$ & S288C genomic DNA & ERF2-F1 and ERF2-R1 \\
\hline DHHC-1 & EST clone (BG437108) & DHHC1-F1 and DHHC1-R1 \\
\hline DHHC-2 & EST clone (CV024712) & DHHC2-F1 and DHHC2-R1 \\
\hline DHHC-3 & HEK $293 \mathrm{~T}$ cDNA & DHHC3-F1 and DHHC3-R1 \\
\hline DHHC-4 & HEK $293 \mathrm{~T}$ cDNA & DHHC4-F1 and DHHC4-R1 \\
\hline DHHC-5 & Human liver cDNA & DHHC5-F1 and DHHC5-R1 \\
\hline DHHC-6 & EST clone (BG395365) & DHHC6-F1 and DHHC6-R1 \\
\hline DHHC-7 & Human liver cDNA & DHHC7-F1 and DHHC7-R1 \\
\hline DHHC-8 & EST clone (BG338434) & DHHC8-F1 and DHHC8-R1 \\
\hline DHHC-9 & Human liver cDNA & DHHC9-F1 and DHHC9-R1 \\
\hline DHHC-10 & EST clone (BG677648) & DHHC10-F1 and DHHC10-R1 \\
\hline DHHC- 11 & EST clone (BI553799) & DHHC11-F1 and DHHC11-R1 \\
\hline
\end{tabular}


DHHC-12 Human liver cDNA

DHHC-13 Human placenta cDNA

DHHC-14 EST clone (BQ687618)

DHHC-15 EST clone (AA424092)

DHHC-16 $\quad$ EST clone (BI544746)

DHHC-17 $\quad$ EST clone (BM698484)

DHHC-18 EST clone (BQ063758)

DHHC-19 EST clone (BG721568)

DHHC-20 EST clone (BI464803)

DHHC-21 Human liver cDNA

DHHC-22 EST clone (BU167563)
DHHC12-F1 and DHHC12-R1

DHHC13-F1 and DHHC13-R1

DHHC14-F1 to F5 and DHHC12-R1

DHHC15-F1 and DHHC15-R1

DHHC16-F1 and DHHC16-R1

DHHC17-F1 and DHHC17-R1

DHHC18-F1 and DHHC18-R1

DHHC19-F1 and DHHC19-R1

DHHC20-F1 and DHHC20-R1

DHHC20-Q1 and DHHC20-Q1c

DHHC20-Q2 and DHHC20-Q2c

DHHC21-F1 and DHHC21-R1

DHHC22-F1 and DHHC22-R1 
Table 3. Primers used in the RT-PCR

Gene

Primer

DHHC-1

DHHC1-F2 and DHHC1-R1

DHHC-2

DHHC2-F2 and DHHC2-R1

DHHC-3

DHHC3-F1 and DHHC3-R1

DHHC-4

DHHC4-F1 and DHHC4-R1

DHHC-5

DHHC5-F2 and DHHC5-R1

DHHC-6

DHHC6-F1 and DHHC6-R1

DHHC-7

DHHC7-F1 and DHHC7-R1

DHHC-8

DHHC8-F2 and DHHC8-R1

DHHC-9

DHHC9-F1 and DHHC9-R1

DHHC-10

DHHC10-F1 and DHHC10-R1

DHHC-11

DHHC11-F1 and DHHC11-R1

DHHC-12

DHHC12-F1 and DHHC12-R1

DHHC-13

DHHC13-F1 and DHHC13-R1

DHHC-14

DHHC14-F6 and DHHC14-R1

DHHC- 15

DHHC15-F2 and DHHC15-R1

DHHC-16

DHHC16-F1 and DHHC16-R1

DHHC-17

DHHC17-F2 and DHHC17-R1

DHHC-18

DHHC18-F2 and DHHC18-R1 
DHHC-19

DHHC-20

DHHC-21

DHHC-22

GAPDH
DHHC19-F1 and DHHC19-R1

DHHC20-F2 and DHHC20-R1

DHHC21-F1 and DHHC21-R1

DHHC22-F2 and DHHC22-R1

GAPDH-F1 and GAPDH-F2 
Table 4. Alternative names and GenBank accession numbers

Protein Alternative name Accession number

\begin{tabular}{|c|c|c|}
\hline Akr1 & Ydr264c & P39010 \\
\hline Akr2 & Yor034c & Q12013 \\
\hline Swf1 & Ydr126w & AY557692 \\
\hline Pfa3 & Ynl326c & P42836 \\
\hline Pfa4 & Yol003c & Q12006 \\
\hline Pfa5 & Ydr459c & Q03289 \\
\hline Erf2 & Y1r246w & Q06551 \\
\hline DHHC-1 & - & NM_013304 \\
\hline DHHC-2 & REAM & BC050272 \\
\hline DHHC-3 & GODZ & NM_016598 \\
\hline DHHC-4 & - & NM_018106 \\
\hline DHHC-5 & - & NM_015457 \\
\hline DHHC-6 & - & BC007213 \\
\hline DHHC-7 & - & NM_017740 \\
\hline DHHC-8 & - & NM_013373 \\
\hline DHHC-9 & - & ВC012826 \\
\hline DHHC-10 & - & NM_024786 \\
\hline DHHC-11 & NIDD & NM_173570 \\
\hline
\end{tabular}




\begin{tabular}{lll} 
DHHC-12 & - & NM_032799 \\
DHHC-13 & - & NM_207340 \\
DHHC-14 & - & NM_024630 \\
DHHC-15 & - & BC103980 \\
DHHC-16 & Aph2 & NM_032327 \\
DHHC-17 & HIP14/AKRL1 & NM_015336 \\
DHHC-18 & - & NM_032283 \\
DHHC-19 & - & NM_144637 \\
DHHC-20 & - & NM_153251 \\
DHHC-21 & - & NM_178566 \\
DHHC-22 & HIP14L/AKRL2 & AB024495 \\
\hline
\end{tabular}


Table 5. Intracellular localization of yeast and human DHHC proteins

\begin{tabular}{|c|c|c|}
\hline Protein & Localization & Reference \\
\hline Akr1 & Golgi & This study, $[3,14]$ \\
\hline Akr2 & Golgi & This study \\
\hline Swf1 & ER & [7] \\
\hline Pfa3 & Vacuole & This study, [6] \\
\hline Pfa4 & ER & This study \\
\hline Pfa5 & Plasma membrane & This study \\
\hline Erf2 & ER & This study, [15] \\
\hline DHHC-1 & ER & This study \\
\hline DHHC-2 & ER/Golgi & This study \\
\hline DHHC-3 & Golgi & This study, [10] \\
\hline DHHC-4 & Golgi & This study \\
\hline DHHC-5 & Plasma membrane & This study \\
\hline DHHC-6 & ER & This study \\
\hline DHHC-7 & Golgi & This study \\
\hline DHHC-8 & Golgi & This study, [29] \\
\hline DHHC-9 & ER/Golgi ${ }^{\mathrm{a}}$ & This study, [12] \\
\hline DHHC-10 & ER & This study \\
\hline DHHC-11 & ER & This study \\
\hline DHHC-12 & ER/Golgi & This study \\
\hline DHHC-13 & ER & This study \\
\hline
\end{tabular}




\begin{tabular}{lll} 
DHHC-14 & ER & This study \\
DHHC-15 & Golgi & This study \\
DHHC-16 & ER & {$[18]$} \\
DHHC-17 & Golgi & This study, [16, 17] \\
DHHC-18 & Golgi & This study \\
DHHC-19 & ER & This study \\
DHHC-20 & Plasma membrane & This study \\
DHHC-21 & Plasma membrane & This study \\
DHHC-22 & ER/Golgi & This study, [16] \\
\hline
\end{tabular}

${ }^{a}$ Although DHHC-9 and DHHC-22 had been reported to be localized in the Golgi apparatus $[12,16]$, we observed here that they are localized both in the ER and in the Golgi. 

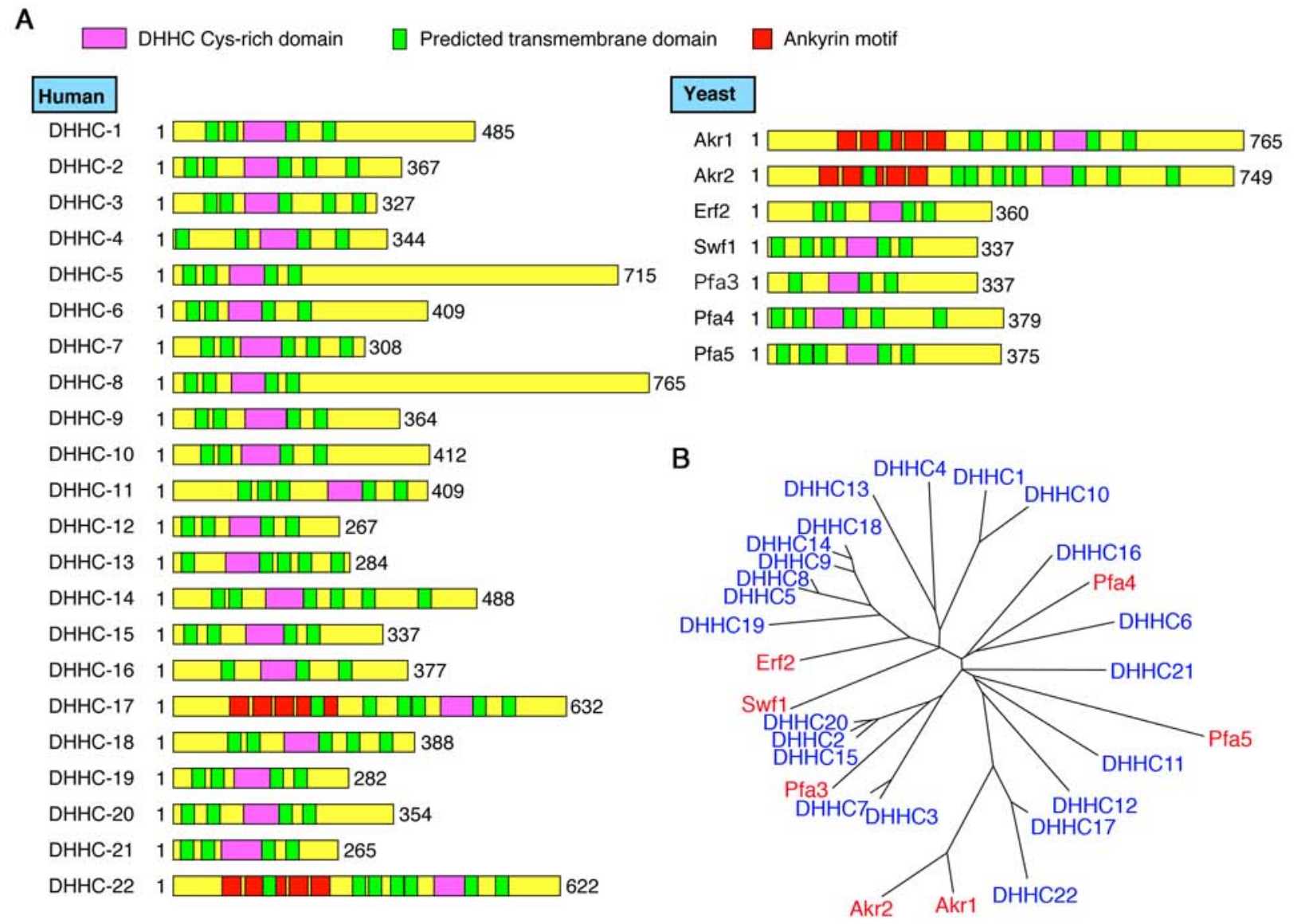


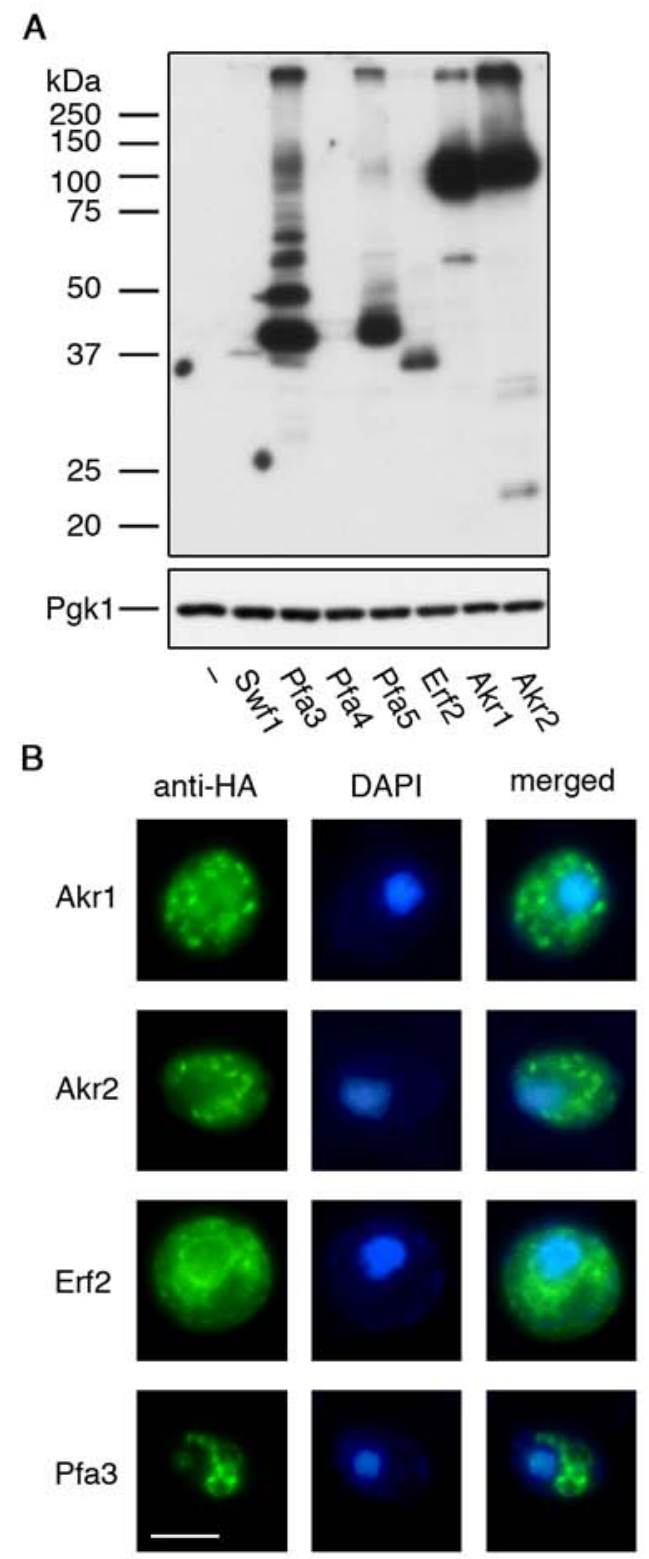

Ohno et al., Fig. 2 
Phase-

EGFP FM4-64 Merged contrast

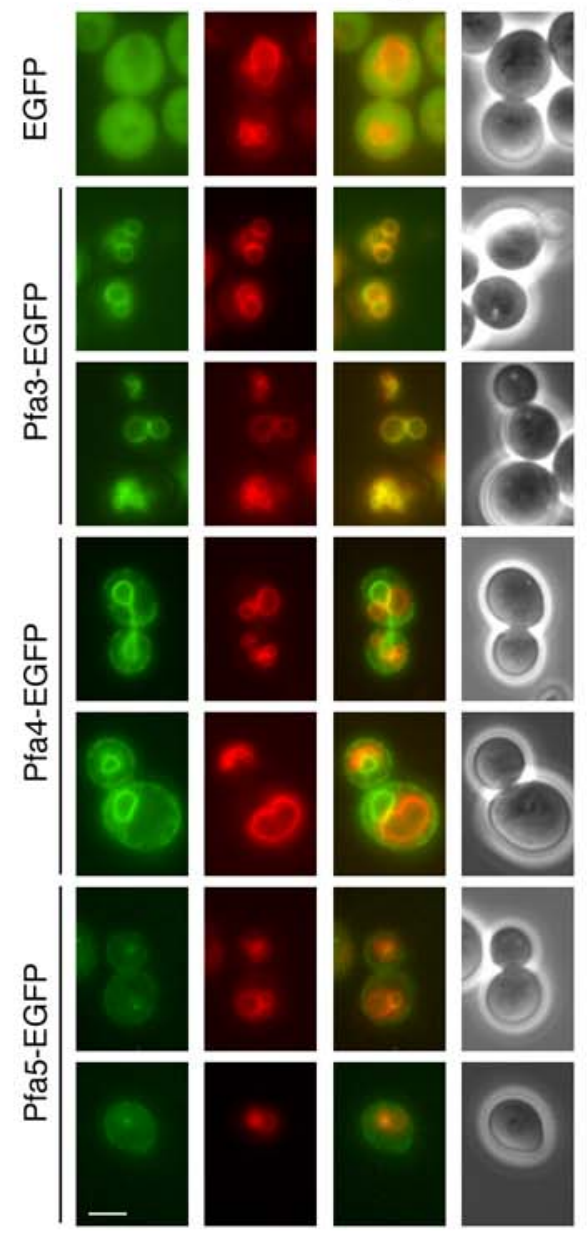

Ohno et al., Fig. 3 

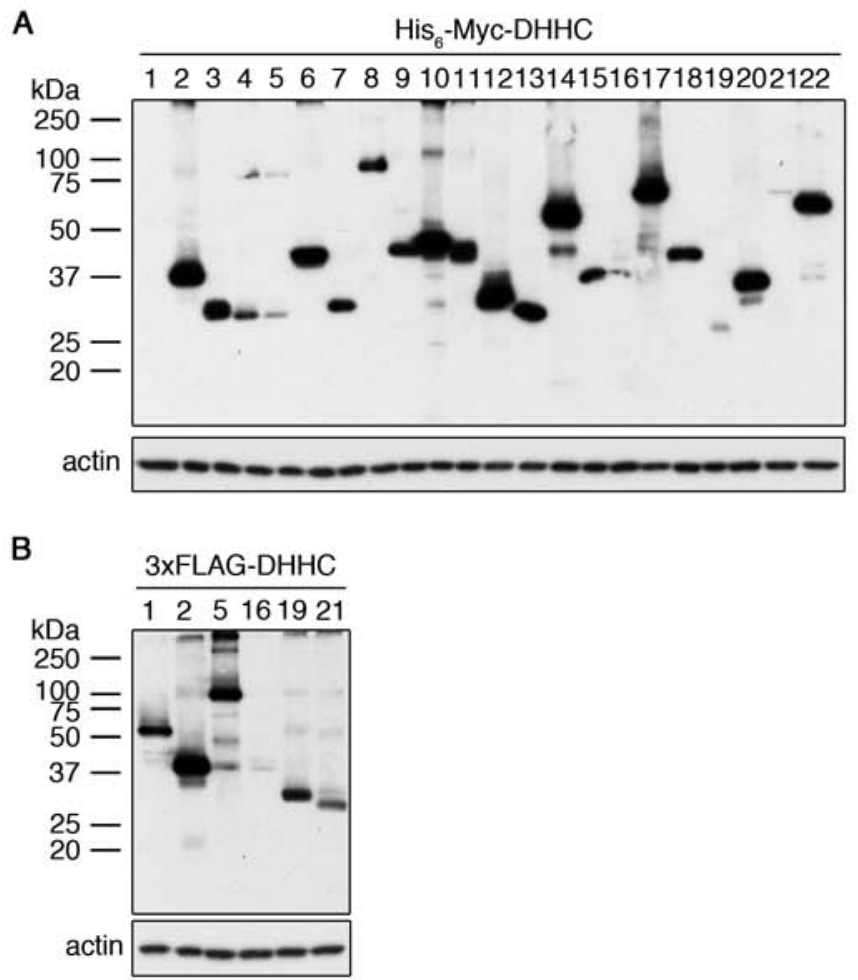

Ohno et al., Fig. 4 

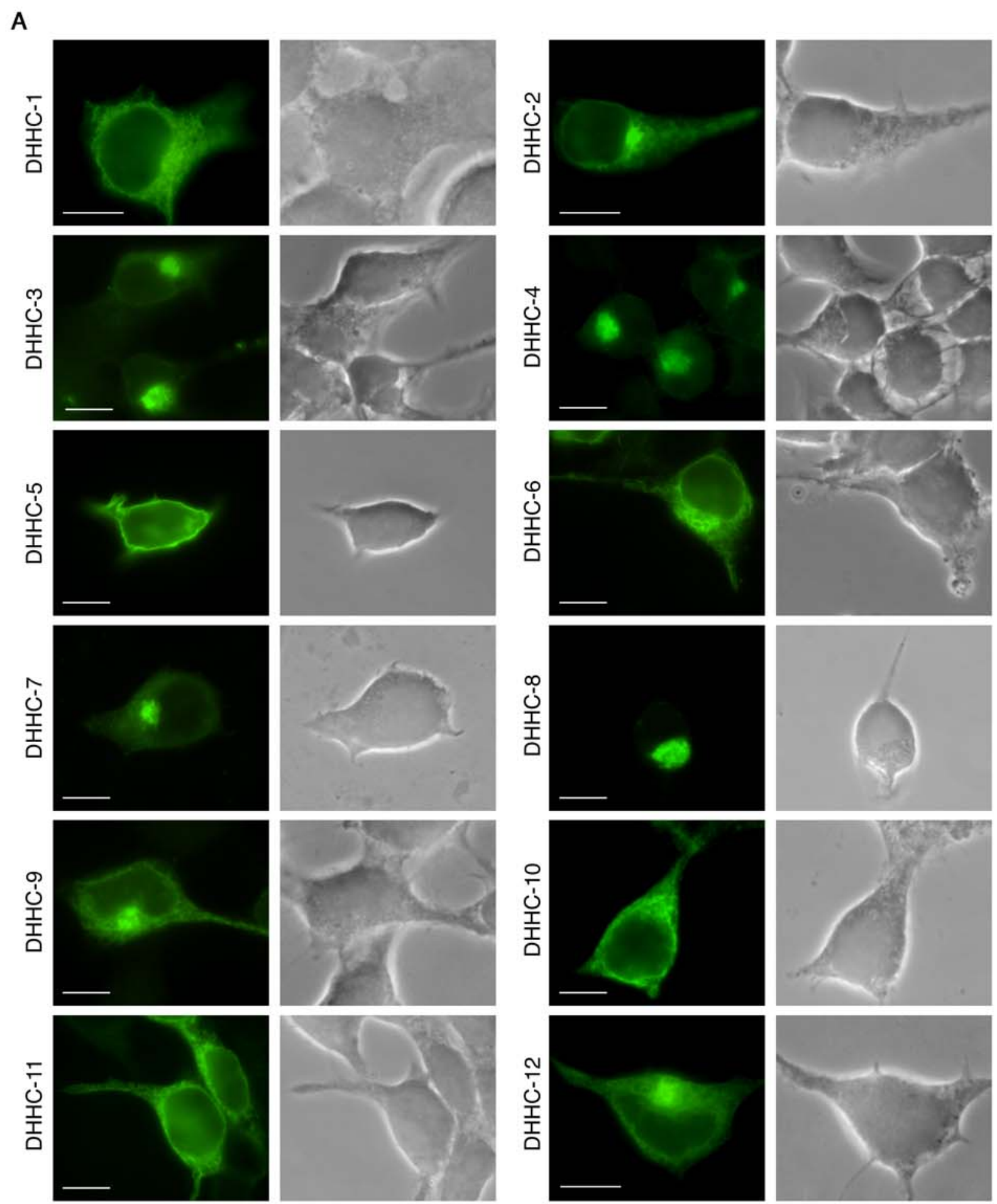

Ohno et al., Fig. 5 

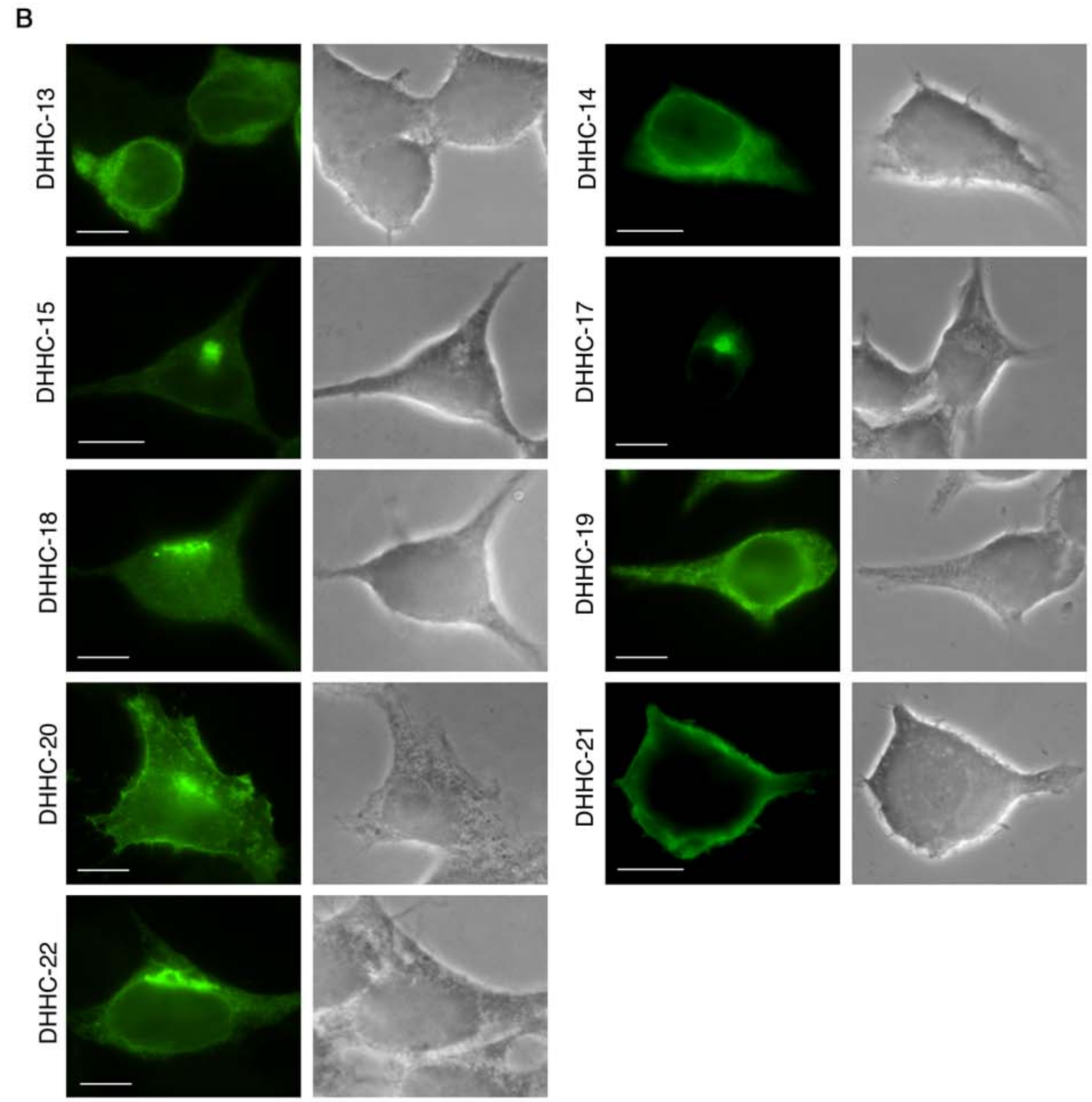

Ohno et al., Fig. 5 


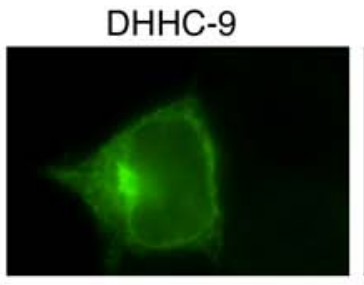

DHHC-9

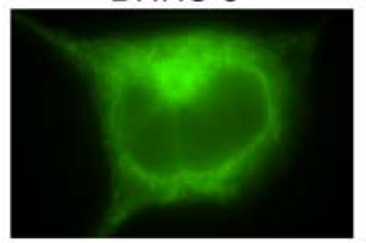

DHHC-11

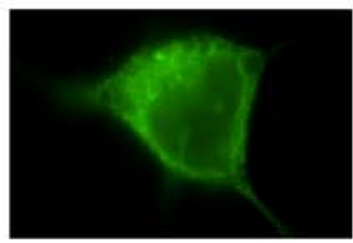

DHHC-12

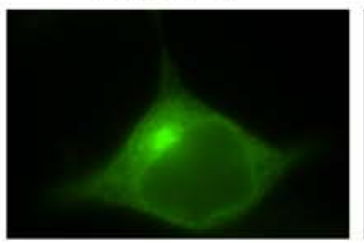

DHHC-12

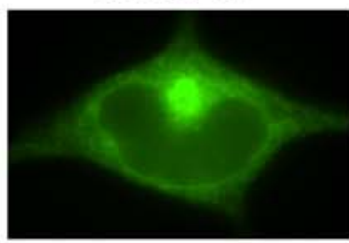

DHHC-15

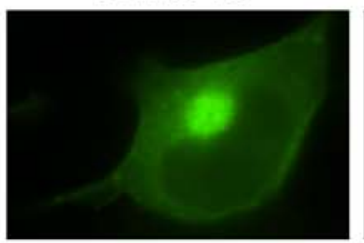

DHHC-22

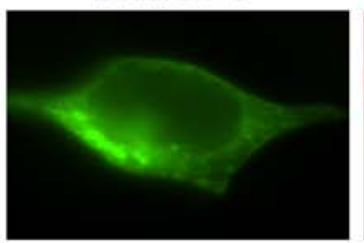

DHHC-22

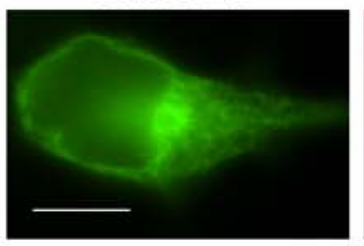

Calreticulin

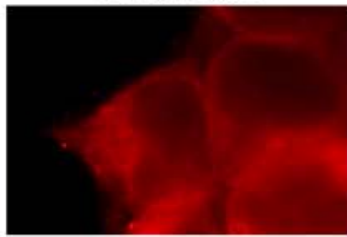

GM130

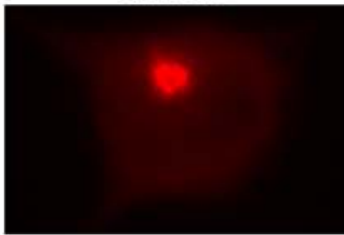

Calreticulin

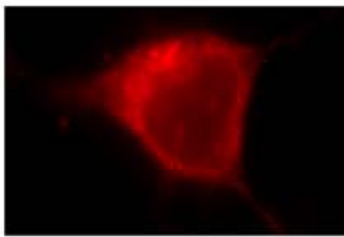

Calreticulin

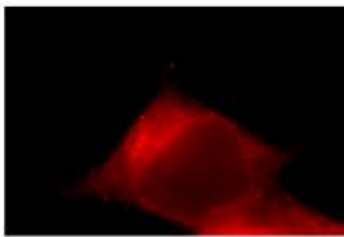

GM130

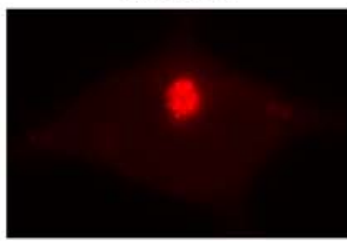

GM130

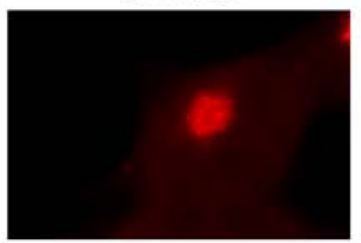

Calreticulin

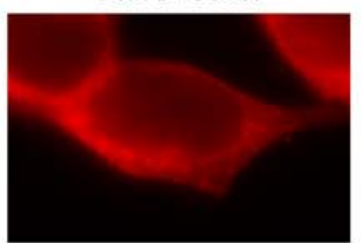

GM130

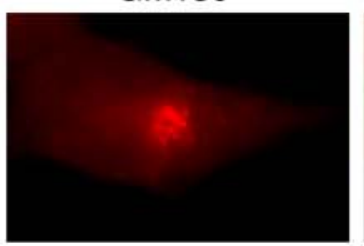

Merged

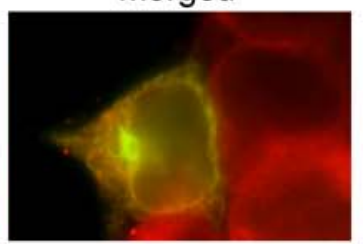

Merged

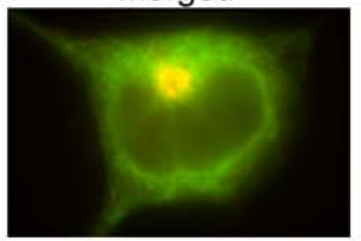

Merged

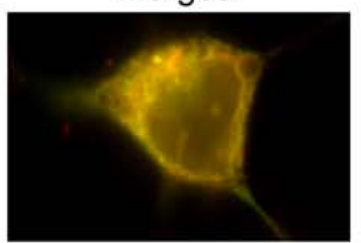

Merged

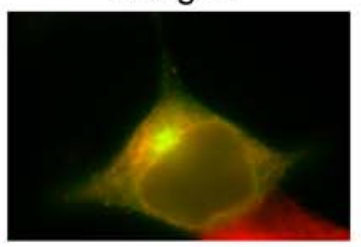

Merged

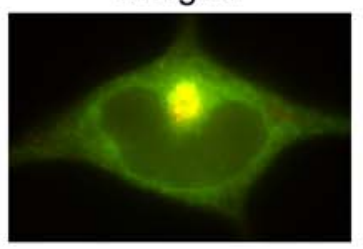

Merged

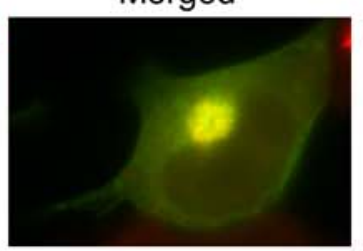

Merged

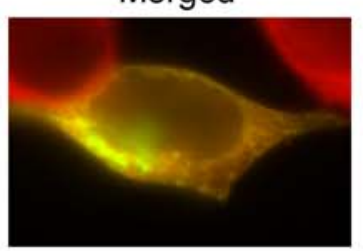

Merged

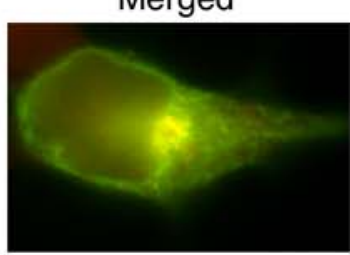

Ohno et al., Fig. 6 


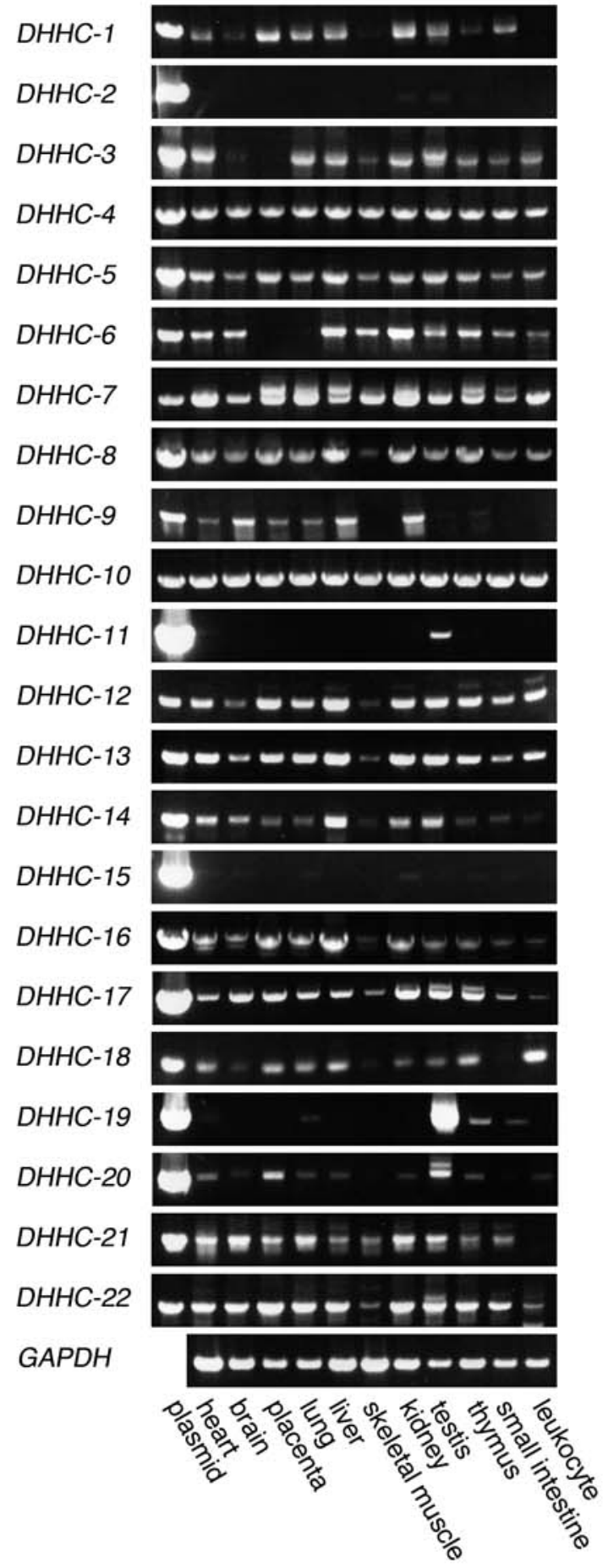

Ohno et al., Fig. 7 